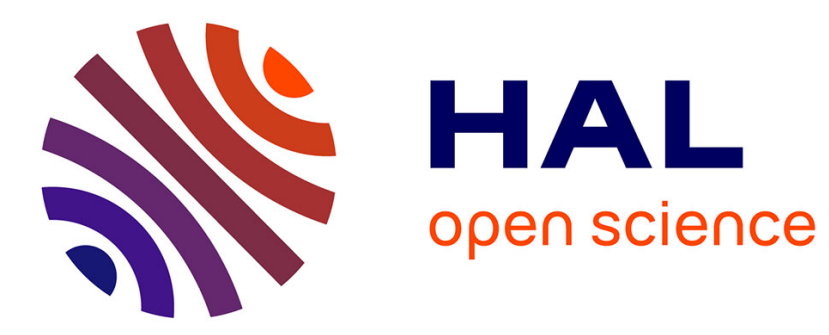

\title{
PHONONS AUX INTERFACES CRISTALLINES
}

\author{
P. Masri, Bahram Djafari-Rouhani
}

\section{To cite this version:}

P. Masri, Bahram Djafari-Rouhani. PHONONS AUX INTERFACES CRISTALLINES. Journal de Physique Colloques, 1984, 45 (C5), pp.C5-37-C5-44. 10.1051/jphyscol:1984504 . jpa-00224112

\section{HAL Id: jpa-00224112 https://hal.science/jpa-00224112}

Submitted on 1 Jan 1984

HAL is a multi-disciplinary open access archive for the deposit and dissemination of scientific research documents, whether they are published or not. The documents may come from teaching and research institutions in France or abroad, or from public or private research centers.
L'archive ouverte pluridisciplinaire HAL, est destinée au dépôt et à la diffusion de documents scientifiques de niveau recherche, publiés ou non, émanant des établissements d'enseignement et de recherche français ou étrangers, des laboratoires publics ou privés. 


\title{
PHONONS AUX INTERFACES CRISTALLINES
}

\author{
P. Masri ${ }^{+}$et B. Djafari-Rouhani ${ }^{*}$ \\ Laboratoire d'Etude des Surfaces, Interfaces et Composants, UR HO 1022 \\ associée au C.N.R.S., U.S.T.L., ELace Eugène Bataizion, \\ 34060 Montpelzier Cedex, France \\ * Laboratoire d'Etude des Surfaces et Interfaces (associé au C.N.R.S.), \\ Faculté Libre des Sciences, I.S.E.N., 13, rue de Toul, \\ 59046 Lizie Cedex, France
}

RESUME :

Notre connaissance des différents modes de vibration aux interfaces cristallines est analysée dans le cadre de la théorie de l'élasticité. L'interface formée par deux cristaux semi-infinis peut supporter des ondes de Stoneley caractérisées par des déplacements décroissants lorsque $I^{\prime}$ on s'éloigne de $I^{\prime}$ interface. Lorsque $l^{\prime}$ on tient compte de 1 'aspect atomique de 1 'interface, nous pouvons prédire de nouvelles structures vibrationnelles. Le cas des milieux isotropes nous permet d'étudier les principales propriétés physiques associēes aux phonons d'interface (vitesse et relation de dispersion ...) et d'avoir des renseignements sur leur condition d'existence.

Une mise au point sur les phonons localisés aux interfaces film-substrat est présentée. Ces études concernent par exemple les modifications de la vitesse des ondes de surface du substrat induites par le film. Comme dans le cas des surfaces, les phonons d'interface peuvent jouer un rôle dans l'ëtude des structures cristallographiques d'interface : dans le cadre de la théorie des phonons mous, le calcul des relations de dispersion des phonons peut nous renseigner sur la stabilité de la structure de 1 'interface considèrée.

\section{ABSTRACT :}

Our knowledge of the different modes of vibration at crystal interfaces is analysed in the framework of the elasticity theory. An interface formed by two semi-infinite crystals can support Stoneley waves which are characterisedbydisplacements which decrease when moving away from both sides of the interface.

When we take into account the atomic character of the interface we can predict new vibrational structures. The case of isotropic media enables us to study the main physical properties associated with the interface phonons (velocity and dispersion relations ...) and to get some information about their conditions of existence.

The studies concerning the phonons localised at film-substrate interfaces are also reviewed. These studies deal for example with the modification of the velocity of the substrate surface waves induced by the film. As in the case of surfaces, interface phonons may be involved in the study of interface crystallographic structures : in the framework of the soft phonons theory, the calculation of phonons dispersion relations enables us to establish whether an interface structure is stable or not.

\section{Introduction}

Les interfaces cristallines présentent un domaine d'application intéressant en Physique des Solides : on peut citer les cas des hétérojonctions, de 1'interface métalsemiconducteur, des systèmes lamellaires métalliques constitués par juxtaposition de films cristallins alternés, des phases adsorbēes, ... Les excitations élémentaires telles que les électrons, les phonons et les magnons ont fait l'objet de nombreuses études.

\footnotetext{
+ Actuellement : Theoretical Physics Division, A.E.R.E., Harwe11, Oxon OXll ORA, $\mathrm{U} . \mathrm{K}$.
} 
L'intérêt pour l'étude de la propagation des ondes élastiques à la surface d'un milieu semi-infini a une longue histoire puisque initialement il était relatif à la propagation des ondes séismiques à la surface de la terre. Ceci s'est maintenant développé en un important domaine de 1a physique des solides où 1 'étude de ces vibrations fournit un moyen efficace pour étudier les propriétés des surfaces et des interfaces. Dans le domaine des petites longueurs d'onde, les phonons sont sensibles à la structure microscopique des surfaces et des interfaces et les mesures des courbes de dispersion associées peuvent justement fournir des informations concernant ces structures et les forces inter-atomiques mises en jeu par les différentes espèces en présence. Lorsqu'ils sont thermiquement excités, les phonons sont source d'entropie /1/ devenant prépondérante dans le domaine des hautes températures et de ce fait pouvant affecter les propriétés des surfaces et des interfaces. Djafari-Rouhani et al. /2/ ont étudié dans le cadre de la théorie des phonons mous le problème des surstructures d'interface. Hoshino /3/ a montré que le crêation de phonons mous à la surface d'un alliage pouvait affecter, comme source d'entropie de vibration, le processus de ségrégation de surface en modifiant l'énergie libre totale du système. Cet effet peut probablement être invoqué dans le cas de ségrégation d'interface.

I1 a été montré /4/ que 1 'étude des propriétés vibrationnelles des surfaces des cristaux ioniques comportant des défauts est essentielle lorsque l'on voulait dêterminer d'une façon self-consistante la structure cristallographique de la surface. C'est le cas de la surface (001) de $\mathrm{MgO}$ comportant du Calcium après ségrégation superficielle de cet élément. Les calculs statiques de la structure de la surface conduisaient à une maille ( $1 \times 1)$ impliquant une relaxation. Les calculs de dynamique de réseau montrent qu'il existe pour cette structure un phonon mou, cette structure vibrationnelle instable disparaissant lorsque 1 'on considère une maille de surface de symétrie différente. Cette méthode de détermination self-consistante de structures cristallographiques de surface doit aussi être mise en oeuvre lorsque l'on cherche à déterminer la structure cristallographique réelle d'une interface.

L'on peut donc dire que dans le domaine des grandes longueurs d'onde un modèle êlastique ne nécessitant pas la connaissance de la structure microscopique de $1^{\prime}$ interface permet d'étudier les principales propriétés physiques des phonons acoustiques d'interface. Dans le domaine des faibles longueurs d'onde, les calculs de dynamique de réseau ne peuvent avoir de réel sens physique sans cette connaissance.

\section{Différents modes d'interface - Modèle élastique isotrope.}

Nous considêrons le système constitué par un film d'épaisseur h déposé sur un substrat supposé semi-infini (Fig. 1). Nous nous intéressons aux différents modes de vibration localisés se propageant parallèlement au plan de 1'interface (direction de propagation $x_{1}$ ). Les ondes considérées sont telles qu'il n'y a pas de variations du champ des déplacements suivant toute direction parallèle à l'interface et perpendiculaire à la direction de propagation. Ces ondes ont donc une phase et une amplitude

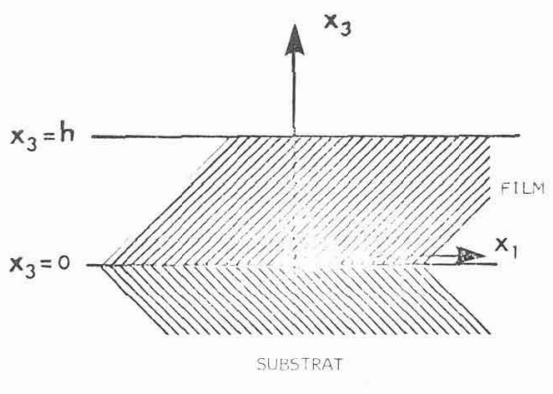

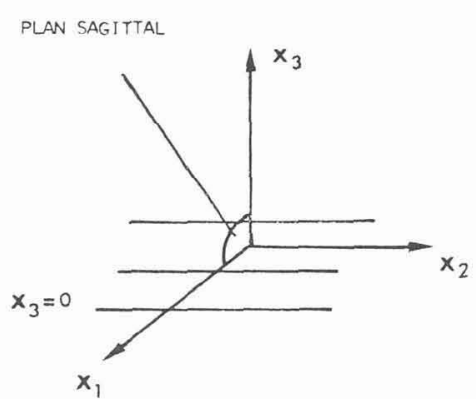


constantes suivant toute ligne parallèle à $x_{2}$ et elles sont inhomogènes parce que leur amplitude décrô̂t lorsque $1^{\prime}$ on s'éloigne de 1 interface et devient nêglịgeable à des distances de l'ordre de la longueur d'onde $\lambda$. Leur qualification à 1 'aide du vecteur $d^{\prime}$ onde $k=2 \pi / \lambda$ se justifie en supposant une périodicitê du système parallèlement au plan de 1 'interface. Ce type de représentation est mis en défaut par la présence êventuelle des défauts d'interface d'autant mieux que 1 'on s'éloigne de la limite des grandes longueurs d'onde.

Dans le cas des matériaux non piézo-êlectriques, les déplacements produits par ces modes d'interface sont solution de l'équation :

$$
\rho \frac{\partial^{2} \mathbf{u}_{\mathbf{j}}}{\partial \mathrm{t}^{2}}=\sum_{\mathbf{i}} \frac{\partial \mathrm{T}_{\mathbf{j}}}{\partial \mathrm{x}_{\mathbf{i}}} \quad i, j=1,2,3
$$

oũ $\mathrm{T}_{j i}$ est $1 \mathrm{le}$ tenseur des contraintes

$$
\mathrm{T}_{j i}=\sum_{1 \mathrm{~m}} \mathrm{C}_{j i l \mathrm{~m}} \frac{\partial \mathrm{u}_{1}}{\partial \mathrm{x}_{\mathrm{m}}}
$$

Cette dernière équation est équivalente à la loi de Hooke reliant les contraintes dans un milieu êlastique aux déformations. On obtient alors :

$$
\rho \frac{\partial^{2} u j}{\partial t^{2}}=\sum_{i l m} C_{i j l m} \frac{\partial^{2} u_{1}}{\partial x_{i} \partial x_{m}}
$$

$C_{i j l m}$ représente le tenseur du module d'élasticité.

Ces équations doivent satisfaire aux conditions aux limites, à savoir :

- Continuité ã 1 'interface du tenseur des contraintes et du champ des dëplacements

- La surface du film côté air ou vide est libre : dans ce cas si $\hat{x}_{3}$ désigne le vecteur unitaire de la normale à la surface, on doit écrire :

d'où les conditions

$$
\mathrm{T}_{\mathrm{i} 3} \hat{\mathrm{x}}_{3}=0 \quad \mathrm{i}=\mathrm{i}, 2,3
$$

$$
\mathrm{T}_{13}=\mathrm{T}_{23}=\mathrm{T}_{33}=0
$$

Dans le cas des milieux isotropes, les éléments du tenseur du mođule d'élasticité non nuls s'écrivent

$$
\begin{aligned}
& c_{11}=c_{22}=c_{33} \\
& c_{12}=c_{13}=c_{23} \\
& c_{44}=c_{55}=c_{66}=\frac{1}{2}\left(c_{11}-c_{12}\right)
\end{aligned}
$$

Dans ce cas il y a découplage des équations d'onde et des équations représentant les conditions aux limites conduisant (fig. 1)

- d'une part à des modes polarisés dans le plan sagittal $\left(x_{1}, x_{3}\right)$ ondes du type Rayleigh par analogie avec les ondes de Rayleigh associees aux surfaces libres.

- d'autre part à des modes du type Love caractérisées par une polarisation transverse $\left(\mathrm{x}_{2}\right)$ perpendiculaire au plan sagittal

\subsection{Modes_du_type_Rayleigh}

Ces modes dérivent des ondes de Rayleigh des surfaces libres perturbées par la création de l'interface. Du point de vue du sens de la perturbation, on peut classer les couples de matériau film-substrat en utilisant le représentation dans le plan

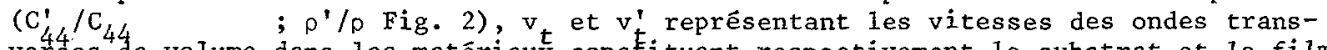
verses de volume dans les matériaux constituant respectivement le substrat et le film. Différentes situations sont à considérer :

\subsubsection{Au-dessus de $1 \mathrm{a}$ droite $v^{\prime}=\sqrt{2} v_{t} ; v^{\prime}>v_{t}$
Pour un tel couple de materiau 1 a présence ${ }_{t}$}

Pour un tel couple de matériau la prétence du film "raidit" les propriétés êlastiques du substrat en produisant une augmentation de la vitesse de l'onde de 


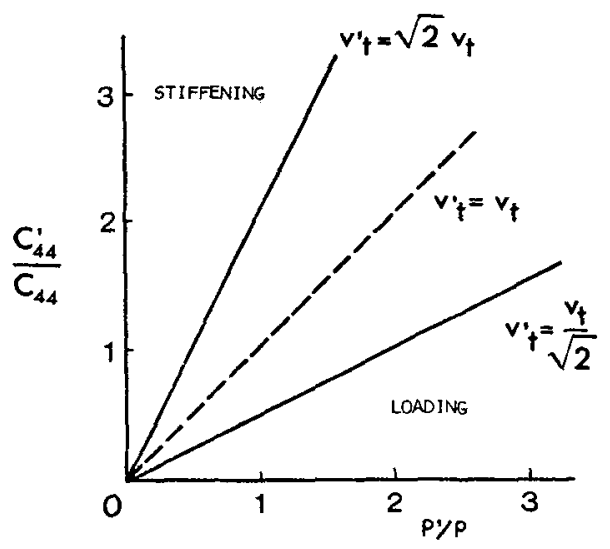

Fig. 2

Rayleigh du substrat libre : par exemple $\mathrm{Si}$ (film)/Zno (substrat). On obtient dans ce cas un seul mode du type Rayleigh existant dàns un domaine réduit de I'épaisseur effective (Fig. 3a). Lorsque 1 'on augmente l'épaisseur du film, sa vitesse évolue de la vitesse de l'onde de Rayleigh du substrat $\left(v_{R}\right)$ jusqu' à $v_{t}$.

Pour les faibles épaisseurs effectives du film les amplitudes des déplacements induits se rapprochent du régime des ondes de Rayleigh des surfaces libres (fig.4). Au voisinage de 1 'épaisseur de coupure, la composante transverse $u_{3}$ décroît lentement en fonction de la distance à l'interface et la composante longitudinale $u_{1}$ est très faible dans le substrat (Fig. 3b). 2.1.2. En-dessous de la droite $v_{t}^{\prime}=v_{t} / \sqrt{2}$;
$v_{t}^{\prime}<v_{t}$

Dans ce cas, Iorsque 1 'on augmente 1 'épaisseur du film la vitesse du mode d'interface décroît à partir de la vitesse de $I^{\prime}$ onde de Rayleigh associée à la surface libre du substrat et tend asymptotiquement vers celle (onde de Rayleigh) de la surface libre du matériau du film : par exemple (Fig. 5) Zno (film)/Si (substrat).

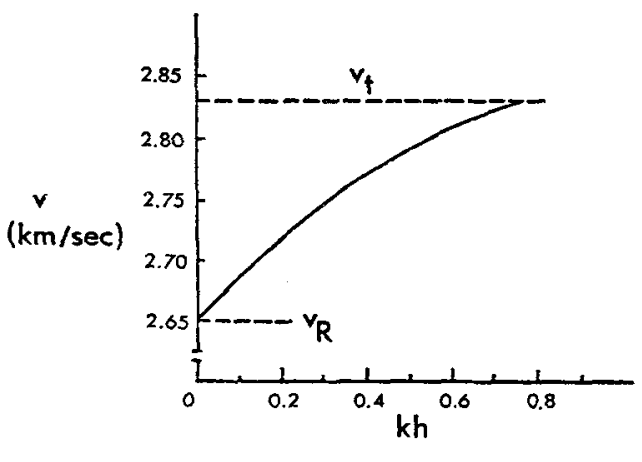

a) Si (film)/Zno (substrat)

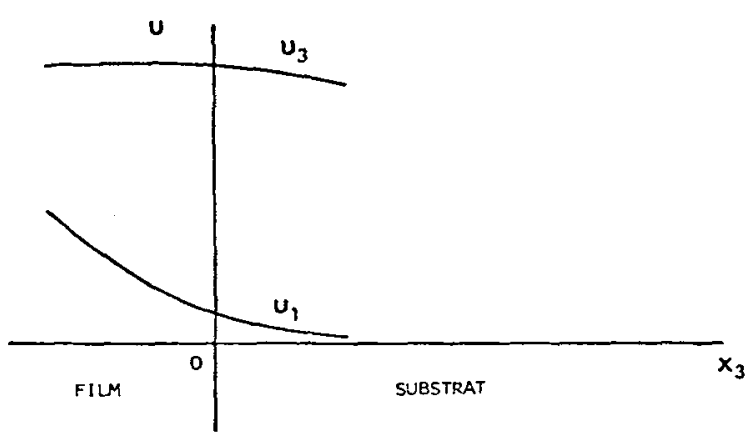

b) Au voisinage de l'épaisseur de coupure

Fig. 3

Pour le système $\mathrm{SiO}_{2} / \mathrm{Si}$ on a

$$
\begin{aligned}
v_{t}(\mathrm{Si}) & =5648 \mathrm{~m} / \mathrm{sec} \\
v_{t}^{\prime}\left(\mathrm{SiO}_{2}\right) & =3705 \mathrm{~m} / \mathrm{sec}
\end{aligned}
$$

Par diffusion Brillouin, Bortolani et al. $/ 6 /$ ont trouvé dans le spectre de diffusion un pic attribué à $I^{\prime}$ 'onde de Rayleigh de $\mathrm{SiO}_{2}$ légèrement modifiée par la présence du substrat $(\mathrm{Si})$.

Pour un tel système, il existe aussi différents modes d'interface appelés modes de Sezawa $15 /$ d'ordre $1,2, \ldots \ldots \ldots$.etc, obtenus en augmentant 1 'épaisseur du film. Ces modes sont des ondes de cisaillement transverses dont la vitesse $v$ est telle que $v_{t}^{\prime}<v<v_{t}$. La figure 5 montre pour le système $\mathrm{ZnO}$ (film)/Si (substrat) la variation ${ }^{t} \mathrm{de} v$ en Eonction de 1 'épaisseur effective du film ainsi que 


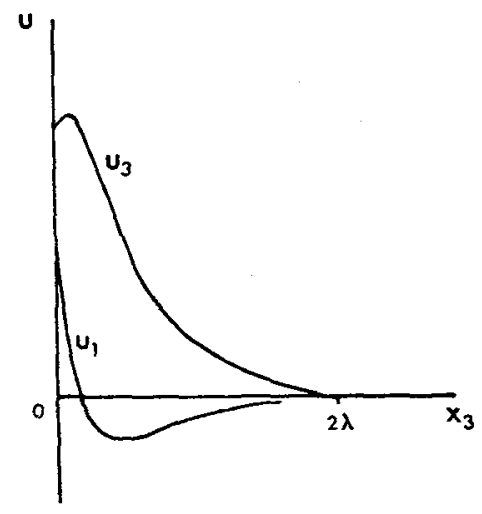

Eig. 4 les valeurs de coupure de cette épaisseur en-dessous de laquelle le mode considéré n'existe plus. Pour le système $\mathrm{SiO}_{2} / \mathrm{Si}$, Bortolani et al. /6/ ont trouve dans le spectre de diffusion Brillouin une structure vibrationnelle large correspondant à un tel mode se propageant dans le film et amorti dans $1 \mathrm{e}$ substrat. Rowe11 et Stegeman /7/ ont trouvé pour le système $\mathrm{SiO}_{2}$ sur substrat semiinfini et par diffusion Brillouin un pic attribue à un mode de Sezawa. La vitesse de ce mode a êté trouvée plus faible que les valeurs calculées en utilisant les constantes élastiques de volume. Les auteurs pensent que ce dêsaccord peut être attribué à des modifications des constantes élastiques du film en cours de fabrication. Aussi la présence de couche de surface défectueuse peut expliquer partiellement cette différence.

En mesurant le spectre de diffusion Brillouin de Al (dépôt de $\simeq 400 \AA$ ) sur $\mathrm{Si}(001)$, Bortolani et al. /8/ trouvent un pic attribué à une onde de Sezawa à une fréquence $\nu=15.9 \mathrm{GHz}$ et se propageant à une vitesse égale à $4367 \mathrm{~m} / \mathrm{sec}$ supérieure à v' (A1) = $2931 \mathrm{~m} / \mathrm{sec}$. Cette onde de Sezawa correspond à une fréquence sensiblement plus basse que celle correspondant à $I^{\prime}$ onde de Rayleigh associée à la surface $(001)$ de $S i \nu_{R}=18.3 \mathrm{GHz}$.

\subsubsection{Domaine voisin de la droite $v_{t}^{\prime} \simeq v_{t}$} Cette situation correspondant au cas où les propriétés êlastiques des deux milieux sont voisines conduit à la présence $\mathrm{d}$ 'ondes du type stoneley /9/ pour deux cristaux semi-infinis. Ces modes produisent des déplacements polarisés dans le plan sagitta1 dont 1 'amplitude décroît exponentiellement lorsque $I^{\prime}$ on s'éloigne de 1 'interface. Les conditions d'existence des ondes de Stoneley, définies sur la figure 6 en fonction des rapports des constantes élastiques des deux milieux et de leurs densités, correspondent au domaine compris entre les courbes 1 et 1 '. Considérons par exemple le cas AI (film)/W (substrat) pour lequel il existe une seule onde du type Rayleigh qui se transforme en onde de Stoneley lorsque 1 'épaisseur du film devient très grande (Fig. 7). On a les conditions d'un raidissement induit par la croissance de I'Aluminium sur le Tungstène : la vitesse de 1 'onde $d$ 'interface varie de $v_{R W} 1 a$ vitesse de 1 'on-

Eig. $5-2 n o(f i z m) / S i$ (substrat) de de Rayleigh du Tungstène pour tendre

asymptotiquement vers la vitesse $v_{\text {de }} I^{\prime}$ 'onde de Stoneley. La situation est différente dans le cas d'un film w sur substrăt Al semi-infini puisqu'elle correspond à un chargement dela surface du substrat. L'on obtient différents modes (Fig. 8)

- Onde de Rayleigh du ler ordre

Sa vitesse, de pente négative aux faibles épaisseurs du film, tend asymptotiquement vers la vitesse de Rayleigh du film.

- Ondes du type Rayleigh d'ordre supérieur

Lorsque I'épaisseur du film augnente, la vitesse de ce type d'onde tenà asymptotiquement vers la vitesse de l'onde de Stoneley. 


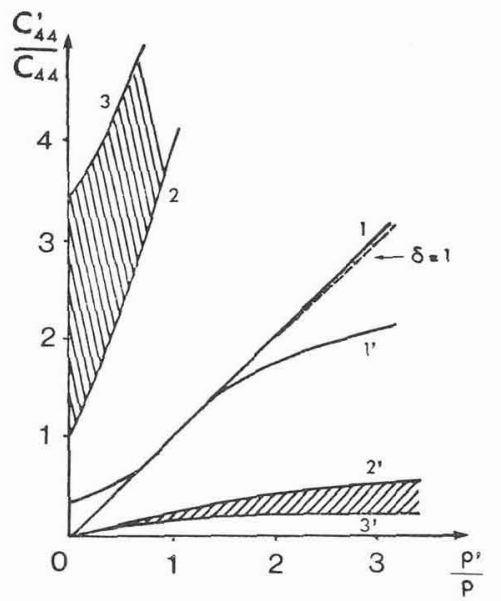

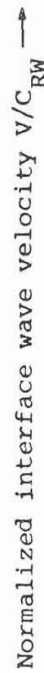

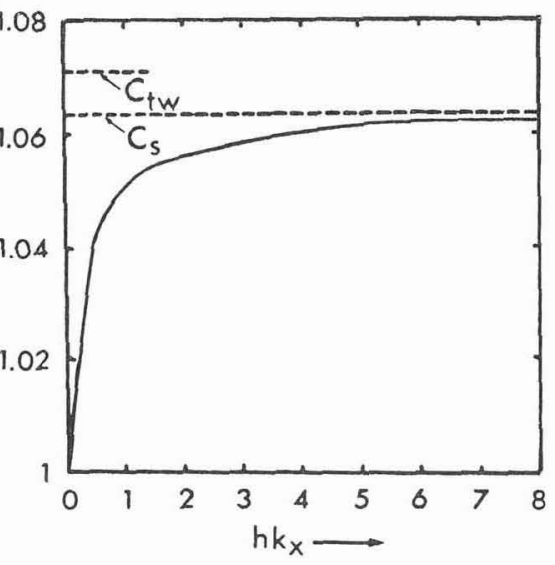

Fig. 6

Régions d'existence de modes localisés et semi-Zocalisés à l'interface de deux matériaux isotropes avec

$$
c_{44} / C_{11}=C_{44}^{\prime} / C_{11}^{\prime}=1 / 3
$$

I'onde de Rayleigh existe entre les courbes 1 et 1'. Dans le reste du plan, il y a un (région non hachurée) ou trois modes semi-localisés (région hachurée).

$$
\delta=C_{t}^{2} / C_{t}^{\prime 2}, v=C_{44} / C_{11}, v^{\prime}=C_{44}^{\prime} / C_{11}^{\prime}
$$

Fig. ? : AZ (film)/W (substrat)

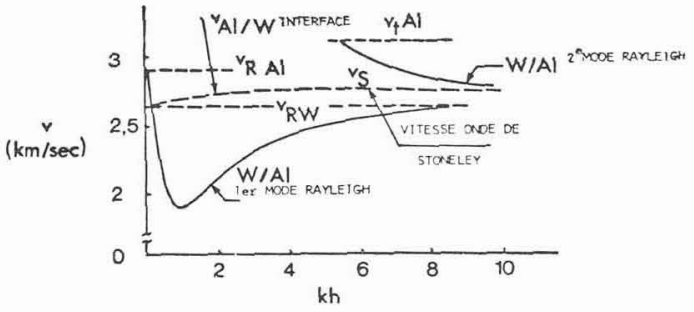

Fig. 8 : W(substrat)/AZ(fizm) 


\subsection{Ondes du_tzype_Love}

Ces ondes existent $s i v_{t}>v_{t}^{\prime}$. Leur polarisation est transverse perpendiculairement au plan sagittal et les déplacements qu'elles produisent décroissent exponentiellement côté substrat et suivent une variation sinusoïdale côté film : 1 'énergie élastique correspondante est donc confinée dans 1 e film. Les courbes de dispersion associées se détachent du bas des bandes transverses du substrat ( $v=v_{t} k_{l}$, Fig. 9) pour différentes valeurs de l'épaisseur effective du film.

A la limite $\mathrm{kh} \rightarrow 0$ la courbe correspondant au mode de Love d'ordre 1 part tangentiellement au bas de bande transverse du substrat. Les autres modes d'ordre $2,3 \ldots$ s'en détachent à mesure que l'épaisseur effective du film augmente (existence de basses fréquences de coupure) et lorsque cette épaisseur devient grande, tous les modes de Love tendent vers la vitesse $v_{t}^{\prime}$ des ondes transversales de volume du film.

I1 existe une thêorie de la diffusion Brillouin par les ondes de Love /9/. Des résultats relatifs à la section efficace de diffusion sont donnés dans le cas d'un substrat de $\mathrm{MgO}$ supportant un film amorphe de $\mathrm{SiO}_{2}: \mathrm{MgO}$ diffusant peu la lumière, ceci permet de supposer que, pratiquement, le processus de diffusion se produit uniquement dans le film. Dans les milieux isotropes au moins il devrait être possible de distinguer ces modes desondes du type Rayleigh, qui correspondent au même domaine de fréquence, puisque ces premiers modifient la polarisation dela lumière incidente.

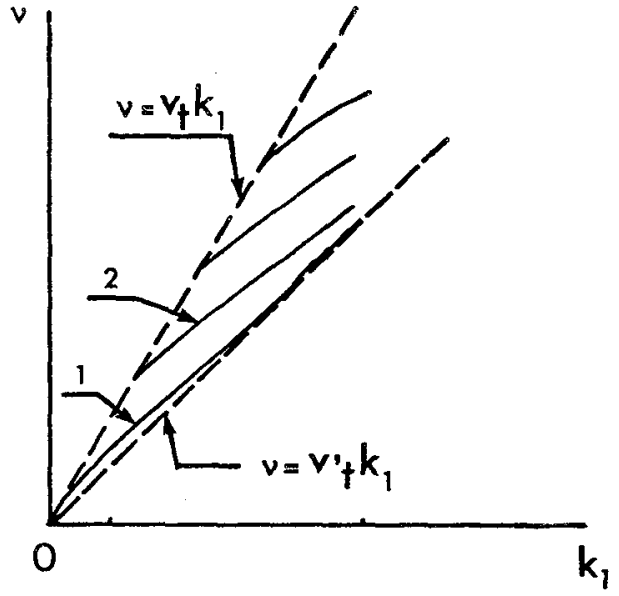

Fig. 9 : Ondes du type Love (d'après la référence 9)
3. Phonons d'interface solide-solide en dehors de la limite elastique

Dans le domaine des faibles longueurs d'onde, les phonons deviennent sensibles à la structure microscopique de 1 'interface : on peut donc en comparant les résultats de modèles théoriques et expérimentaux en déduire des informations sur l'interface (géométrie, relaxation, interactions à 1 'interface). L'étude de modèles atomiques permet par ailleurs d'obtenir des structures vibrationnelles nouvelles non mises en évidence par l'étude des phonons d'interface dans le cadre de la théorie de l'élasticité. Benedek et Velasco /10/ ont étudié les phonons d'interface entre deux cristaux ioniques LiF/KF (001) pour lesquels les paramètres cristallins sont dans un rapport égal à $3 / 4$. D'une façon schêmatique, 1 'on peut dire que KF se trouve donc en contact avec un cristal plus léger. De ce fait les fréquences de vibration dans KF seront augmentées, celles dans LiF seront abaíssées : ainsi le mode de Rayleigh de KF se transforme en un mode de Stoneley et le mode de Rayleigh de LiF donne un mode résonant fortement mélangé aux autres modes de KF. (Fig. 10 par exemple, réf. 10, courbe

On obtient aussi deux modes fortement résonants (Fig. 10, Courbes $I_{1}$ et $I_{2}$ ) issus des branches acoustiques et optiques transverses de KF par interaction avec les bandes de $\mathrm{LiF}$. Le mode de Lucas de LiF (courbe $\mathrm{LM}_{1}$ ) est pratiquement non perturbé en énergie car il est situé à des fréquences élevées telles qu'il ne peut se propager dans KF. 


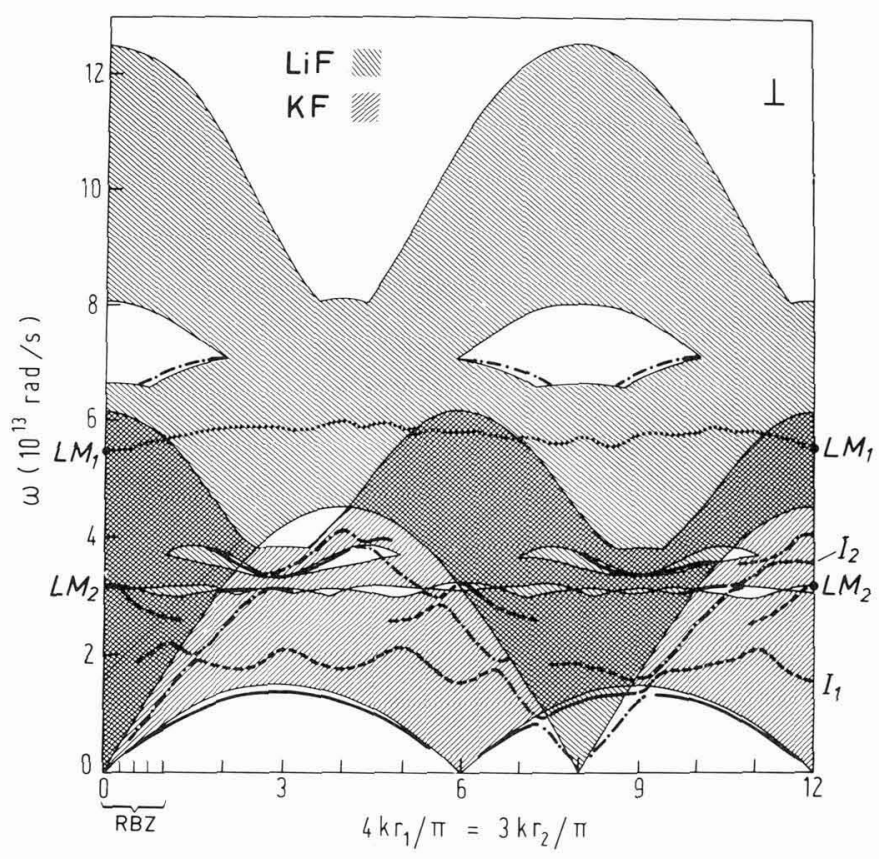

Eig. 10 - D'après la référence 10 avec la permission des auteurs

\section{Références}

1. EWING R.H., Acta Metallurgica, 19, (1971), 1359.

2. DJAFARI-ROUHANI B., DOBRZYNSKI I. et MASRI P., Surface Science, $\underline{52},(1975), 29$.

3. HOSHINO K., J. Phys. Soc., Jpn., 50 (1981) 577.

4. MASRI P. et TASKER P.W., V International Conference on Solid Surfaces, Madrid(1983)

5. SEZAWA K. et KANAI K., Bu11. Earthquake Res. Inst. (Tokyo), I3 (1935) 471.

6. BORTOZANI V., NIZZOLI F., SANTORO G, et SANDERCOCK J.R., Phys. Rev. B25 (1982) 3442.

7. ROWELL N.L. et STEGEMAN G.I. , Phys. Rev. Lett., 41 (1978) 970.

8. BORTOLANI Y., NIZZOLI F., SANTORO G., MARWIN A. et SANDERCOCK J.R., Phys. Rev. Lett., 43 (I979) 224.

9. ALBUQUERQUE E.L., LOUDON R, et TILLEY D.R., J. Phys. C Solid St. Phys., 13 (1980) 1775.

10. BENEDEK G, et VELASCO V., Phys. Rev. B23 (1981) 6691. 